\title{
Nominata de pareceristas (2019 e 2020)
}

\begin{tabular}{|c|c|c|}
\hline Parecerista & Titulação & Filiação Institucional \\
\hline Aline Iubel & Doutorado em Antropologia Social & $\begin{array}{l}\text { Universidade Estadual de Campinas } \\
\text { (UNICAMP) }\end{array}$ \\
\hline $\begin{array}{l}\text { Alline Torres Dias da } \\
\text { Cruz }\end{array}$ & Doutorado em Antropologia Social & Colégio Pedro II - RJ \\
\hline Ana Luisa Fayet Sallas & Doutorado em História & Universidade Federal do Paraná (UFPR) \\
\hline $\begin{array}{l}\text { Ana Paula Massadar } \\
\text { Morel }\end{array}$ & Doutorado em Antropologia Social & Universidade Federal Fluminense (UFF) \\
\hline Andressa Lewandowski & Doutorado em Antropologia Social & $\begin{array}{l}\text { Universidade da Integração Internacional } \\
\text { da Lusofonia Afro-Brasileira (UNILAB) }\end{array}$ \\
\hline Aristóteles Barcelos Neto & $\begin{array}{l}\text { Doutorado em Ciência Social } \\
\text { (Antropologia Social) }\end{array}$ & University of East Anglia \\
\hline Cauê Krüger & $\begin{array}{l}\text { Doutorado em Sociologia e } \\
\text { Antropologia }\end{array}$ & $\begin{array}{l}\text { Pontifícia Universidade Católica do } \\
\text { Paraná (PUC-PR) }\end{array}$ \\
\hline Christine Escallier & Doutorado em Ciências Humanas & Universidade da Madeira, Portugal \\
\hline Ciméa Bevilaqua & $\begin{array}{l}\text { Doutorado em Ciência Social } \\
\text { (Antropologia Social) }\end{array}$ & Universidade Federal do Paraná (UFPR) \\
\hline Daniel Belik & Doutorado em Antropologia Social & $\begin{array}{l}\text { Universidade Federal de Rondônia } \\
\text { (UNIR) }\end{array}$ \\
\hline Daniel de Lucca R. Costa & Doutorado em Ciências Sociais & $\begin{array}{l}\text { Universidade da Integração Internacional } \\
\text { da Lusofonia Afro-Brasileira (UNILAB) }\end{array}$ \\
\hline Denise Fajardo Grupioni & $\begin{array}{l}\text { Doutorado em Ciência Social } \\
\text { (Antropologia Social) }\end{array}$ & $\begin{array}{l}\text { Instituto Iepé de Pesquisa e Formação } \\
\text { Indígena (IEPÉ) }\end{array}$ \\
\hline Diego Villar & $\begin{array}{l}\text { Doutorado em Filosofia e Letras } \\
\text { com orientação em Antropologia }\end{array}$ & Universidade de Buenos Aires \\
\hline Edilene Coffaci de Lima & $\begin{array}{l}\text { Doutorado em Ciência Social } \\
\text { (Antropologia Social) }\end{array}$ & Universidade Federal do Paraná (UFPR) \\
\hline
\end{tabular}




\begin{tabular}{|c|c|c|}
\hline Edward MacRae & $\begin{array}{l}\text { Doutorado em Ciência Social } \\
\text { (Antropologia Social) }\end{array}$ & Universidade Federal da Bahia (UFBA) \\
\hline Erica Quinaglia & $\begin{array}{l}\text { Doutorado em Sociologia, } \\
\text { Demografia e Antropologia }\end{array}$ & $\begin{array}{l}\text { Universidade Federal do Pará (UFOPA) / } \\
\text { Universidade de Brasília (UnB) }\end{array}$ \\
\hline Eva Lenita Scheliga & $\begin{array}{l}\text { Doutorado em Ciência Social } \\
\text { (Antropologia Social) }\end{array}$ & Universidade Federal do Paraná (UFPR) \\
\hline Eveline Stella de Araújo & Doutorado em Saúde Pública & Universidade Federla do Paraná (UFPR) \\
\hline Éverton Luiz Pereira & Doutorado em Antropologia Social & Universidade de Brasília (UnB) \\
\hline Fabiana Maizza & $\begin{array}{l}\text { Doutorado em Ciência Social } \\
\text { (Antropologia Social) }\end{array}$ & $\begin{array}{l}\text { Universidade Federal do Pernambuco } \\
\text { (UFPE) }\end{array}$ \\
\hline $\begin{array}{l}\text { Fernanda Azeredo de } \\
\text { Moraes }\end{array}$ & $\begin{array}{l}\text { Doutoranda em Antropologia e } \\
\text { Etnologia }\end{array}$ & $\begin{array}{l}\text { École des Hautes Études en Sciences } \\
\text { Sociales (EHESS - Paris) }\end{array}$ \\
\hline Flávio Wiik & Doutorado em Antropologia & $\begin{array}{l}\text { Universidade Estadual de Londrina } \\
\text { (UEL) }\end{array}$ \\
\hline Indira Nahomi Viana & Doutorado em Antropologia Social & Universidade Federal de Goiás (UFG) \\
\hline Jean Segata & Doutorado em Antropologia Social & $\begin{array}{l}\text { Universidade Federal do Rio Grande do } \\
\text { Sul (UFRGS) }\end{array}$ \\
\hline João Kleba Lisboa & Doutorado em Antropologia Social & Pesquisador independente \\
\hline João Rickli & Doutorado em Antropologia Social & Universidade Federal do Paraná (UFPR) \\
\hline Jorge Eremites de Oliveira & Doutorado em História & Universidade Federal de Pelotas (UFPel) \\
\hline José Glebson Vieira & $\begin{array}{l}\text { Doutorado em Ciência Social } \\
\text { (Antropologia Social) }\end{array}$ & $\begin{array}{l}\text { Universidade Federal do Rio Grande do } \\
\text { Norte (UFRN) }\end{array}$ \\
\hline Juliana M. Soares Campos & Doutorado em Antropologia & $\begin{array}{l}\text { Universidade Federal de Minas Gerais } \\
\text { (UFMG) }\end{array}$ \\
\hline Juliane Bazzo & Doutorado em Antropologia Social & $\begin{array}{l}\text { Universidade Federal da Grande } \\
\text { Dourados (UFGD) }\end{array}$ \\
\hline Karina Biondi & Doutorado em Antropologia Social & $\begin{array}{l}\text { Universidade Estadual do Maranhão } \\
\text { (UEMA) }\end{array}$ \\
\hline Lailson Ferreira da Silva & Doutorado em Ciências Sociais & $\begin{array}{l}\text { Universidade da Integração Internacional } \\
\text { da Lusofonia Afro-Brasileira (UNILAB) }\end{array}$ \\
\hline Laura Pérez Gil & Doutorado em Antropologia Social & Universidade Federal do Paraná (UFPR) \\
\hline
\end{tabular}




\begin{tabular}{|c|c|c|}
\hline $\begin{array}{l}\text { Leonardo Campoy } \\
\text { Carbonieri }\end{array}$ & Doutorado em Antropologia & $\begin{array}{l}\text { Pontifícia Universidade Católica do } \\
\text { Paraná (PUC-PR) }\end{array}$ \\
\hline Leonardo Ribeiro da Cruz & Doutorado em Sociologia & Universidade Federal do Pará (UFPA) \\
\hline Levi Marques Pereira & $\begin{array}{l}\text { Doutorado em Ciência Social } \\
\text { (Antropologia Social) }\end{array}$ & $\begin{array}{l}\text { Universidade Federal da Grande } \\
\text { Dourados (UFGD) }\end{array}$ \\
\hline Lígia Duque Platero & Doutorado em Ciências Humanas & Universidade Federal Fluminense (UFF) \\
\hline Lorenzo Macagno & Doutorado em Ciências Humanas & Universidade Federal do Paraná (UFPR) \\
\hline Luisa Elvira Belaunde & Doutorado em Antropologia & $\begin{array}{l}\text { Museu Nacional/Universidade Federal } \\
\text { do Rio de Janeiro (UFRJ) }\end{array}$ \\
\hline Manuel Caleiro & $\begin{array}{l}\text { Doutorado em Direito } \\
\text { Socioambiental }\end{array}$ & $\begin{array}{l}\text { Universidade Federal do Mato Grosso do } \\
\text { Sul (UFMS) }\end{array}$ \\
\hline Marcos Silveira & Doutorado em Antropologia Social & Universidade Federal do Paraná (UFPR) \\
\hline Maria Inês S. Borges & Doutorado em Antropologia Social & Universidade Federal do Paraná (UFPR) \\
\hline Martina Ahlert & Doutorado em Antropologia Social & $\begin{array}{l}\text { Universidade Federal do Maranhão } \\
\text { (UFMA) }\end{array}$ \\
\hline Mércia R. Rangel Batista & Doutorado em Antropologia Social & $\begin{array}{l}\text { Universidade Federal de Campina } \\
\text { Grande (UFCG) }\end{array}$ \\
\hline Miguel Carid Naveira & Doutorado em Antropologia Social & Universidade Federal do Paraná (UFPR) \\
\hline Patrícia Carvalho Rosa & Doutorado em Antropologia Social & Instituto Mamirauá \\
\hline Paulo Renato Guérios & Doutorado em Antropologia Social & Universidade Federal do Paraná (UFPR) \\
\hline Philippe Erikson & Doutorado em Etnologia & Universidade de Paris X-Nanterre \\
\hline $\begin{array}{l}\text { Priscila Tavares dos } \\
\text { Santos }\end{array}$ & Doutorado em Antropologia Social & Universidade Federal Fluminense (UFF) \\
\hline Ricardo Cid Fernandes & $\begin{array}{l}\text { Doutorado em Ciência Social } \\
\text { (Antropologia Social) }\end{array}$ & Universidade Federal do Paraná (UFPR) \\
\hline Rodrigo Toniol & Doutorado em Antropologia Social & $\begin{array}{l}\text { Universidade Estadual de Campinas } \\
\text { (Unicamp) }\end{array}$ \\
\hline Romulo Labronici & Doutorado em Antropologia & Universidade Federal Fluminense (UFF) \\
\hline $\begin{array}{l}\text { Rosa Virginia A. de A. } \\
\text { Melo }\end{array}$ & Doutorado em Antropologia Social & Pesquisadora independente \\
\hline
\end{tabular}




\begin{tabular}{l|l|l}
\hline Rubia Carla F. Giordani & Doutorado em Sociologia & Universidade Federal do Paraná (UFPR) \\
\hline Sandra Stoll & $\begin{array}{l}\text { Doutorado em Ciência Social } \\
\text { (Antropologia Social) }\end{array}$ & Universidade Federal do Paraná (UFPR) \\
\hline Sheila Cavalcante & Doutoranda em Antropologia & Universidade Federal do Paraná (UFPR) \\
\hline Simone Becker & Doutorado em Antropologia Social & $\begin{array}{l}\text { Universidade Federal da Grande } \\
\text { Dourados (UFGD) }\end{array}$ \\
\hline Valéria Macedo & $\begin{array}{l}\text { Doutorado em Ciência Social } \\
\text { (Antropologia Social) }\end{array}$ & $\begin{array}{l}\text { Universidade Federal de São Paulo } \\
\text { (UNIFESP) }\end{array}$ \\
\hline
\end{tabular}




\section{Nominata dos pareceristas - Dossiê Etnografias em contextos pedagógicos}

\begin{tabular}{|c|c|c|}
\hline Parecerista & Titulação & Filiação institucional \\
\hline $\begin{array}{l}\text { Alexandra E. Vieira } \\
\text { Alencar }\end{array}$ & $\begin{array}{l}\text { Doutorado em } \\
\text { Antropologia Social }\end{array}$ & Universidade Federal de Santa Catarina (UFSC) \\
\hline Alexandre Barbosa Pereira & $\begin{array}{l}\text { Doutorado em Ciência } \\
\text { Social (Antropologia } \\
\text { Social) }\end{array}$ & Universidade Federal de São Paulo (UNIFESP) \\
\hline Alexandre Zarias & Doutorado em Sociologia & Fundação Joaquim Nabuco (Fundaj) \\
\hline Aline Castilho Crespe & Doutorado em História & $\begin{array}{l}\text { Universidade Federal da Grande Dourados } \\
\text { (UFGD) }\end{array}$ \\
\hline $\begin{array}{l}\text { Ana Gretel E. } \\
\text { Böschemeier }\end{array}$ & $\begin{array}{l}\text { Doutorado em } \\
\text { Antropologia Social }\end{array}$ & $\begin{array}{l}\text { Universidade Federal do Rio Grande do Norte } \\
\text { (UFRN) }\end{array}$ \\
\hline Ana Letícia de Fiori & $\begin{array}{l}\text { Doutorado em Ciência } \\
\text { Social (Antropologia } \\
\text { Social) }\end{array}$ & Universidade Federal do Acre (UFAC) \\
\hline Ana Paula Alves Ribeiro & $\begin{array}{l}\text { Doutorado em Saúde } \\
\text { Coletiva }\end{array}$ & $\begin{array}{l}\text { Universidade do Estado do Rio de Janeiro } \\
\text { (UERJ) }\end{array}$ \\
\hline Ana Pires do Prado & $\begin{array}{l}\text { Doutorado em } \\
\text { Antropologia Social e } \\
\text { Cultural }\end{array}$ & Universidade Federal do Rio de Janeiro (UFRJ) \\
\hline $\begin{array}{l}\text { André L. Guerreiro } \\
\text { Oliveira }\end{array}$ & Doutorado em Educação & $\begin{array}{l}\text { Secretaria de Educação do Estado do Paraná } \\
\text { (SEED/PR) }\end{array}$ \\
\hline Andréia Baia Prestes & $\begin{array}{l}\text { Doutorado em } \\
\text { Antropologia Social }\end{array}$ & Federação do Povo Huni Kui do Acre (FEPHAC) \\
\hline $\begin{array}{l}\text { Anelise dos Santos } \\
\text { Gutterres }\end{array}$ & $\begin{array}{l}\text { Doutorado em } \\
\text { Antropologia Social }\end{array}$ & Universidade Federal do Rio de Janeiro (UFRJ) \\
\hline $\begin{array}{l}\text { Ângela M. Scalabrin } \\
\text { Coutinho }\end{array}$ & $\begin{array}{l}\text { Doutorado em Estudos da } \\
\text { Criança }\end{array}$ & Universidade Federal do Paraná (UFPR) \\
\hline $\begin{array}{l}\text { Amanda Rodrigues } \\
\text { Marqui }\end{array}$ & $\begin{array}{l}\text { Doutorado em } \\
\text { Antropologia Social }\end{array}$ & Universidade Federal de São Carlos (UFSCAR) \\
\hline Ari Jose Sartori & $\begin{array}{l}\text { Doutorado em } \\
\text { Antropologia Social }\end{array}$ & Universidade Federal da Fronteira Sul (UFFS) \\
\hline Barbara de Souza Fontes & $\begin{array}{l}\text { Doutorado em } \\
\text { Antropologia }\end{array}$ & Colégio Pedro II \\
\hline
\end{tabular}




\begin{tabular}{|c|c|c|}
\hline $\begin{array}{l}\text { Carlos A. B. Plínio dos } \\
\text { Santos }\end{array}$ & $\begin{array}{l}\text { Doutorado em } \\
\text { Antropologia }\end{array}$ & Universidade de Brasília (UnB) \\
\hline Cauê Krüger & $\begin{array}{l}\text { Doutorado em } \\
\text { Antropologia Social }\end{array}$ & $\begin{array}{l}\text { Pontifícia Universidade Católica do Paraná } \\
\text { (PUC/PR) }\end{array}$ \\
\hline Chantal Victória Medaets & Doutorado em Educação & Universidade Estadual de Campinas (UNICAMP) \\
\hline Delma Pessanha Neves & $\begin{array}{l}\text { Doutorado em } \\
\text { Antropologia Social }\end{array}$ & Universidade Federal Fluminense (UFF) \\
\hline Diana Milstein & $\begin{array}{l}\text { Doutorado em } \\
\text { Antropologia Social }\end{array}$ & $\begin{array}{l}\text { Universidad Nacional del Comahue / Instituto de } \\
\text { Desarrollo Económico y Social (IDES) }\end{array}$ \\
\hline Diógenes Egídio Cariaga & $\begin{array}{l}\text { Doutorado em } \\
\text { Antropologia Social }\end{array}$ & $\begin{array}{l}\text { Universidade Estadual do Mato Grosso do Sul } \\
\text { (UEMS) }\end{array}$ \\
\hline Edilma do N. J. Monteiro & $\begin{array}{l}\text { Doutorado em } \\
\text { Antropologia Social }\end{array}$ & $\begin{array}{l}\text { Universidade Federal do Rio Grande do Norte } \\
\text { (UFRN) }\end{array}$ \\
\hline Esmael Alves de Oliveira & $\begin{array}{l}\text { Doutorado em } \\
\text { Antropologia Social }\end{array}$ & $\begin{array}{l}\text { Universidade Federal da Grande Dourados } \\
\text { (UFGD) }\end{array}$ \\
\hline Flávia Ferreira Pires & $\begin{array}{l}\text { Doutorado em } \\
\text { Antropologia Social }\end{array}$ & Universidade Federal da Paraíba (UFPB) \\
\hline $\begin{array}{l}\text { Florencio Rekayg } \\
\text { Fernandes }\end{array}$ & $\begin{array}{l}\text { Doutorando em } \\
\text { Antropologia Social }\end{array}$ & $\begin{array}{l}\text { Secretaria de Educação do Estado do Paraná } \\
\text { (SEED/PR) }\end{array}$ \\
\hline $\begin{array}{l}\text { Francisca Navantino P. de } \\
\text { Angelo }\end{array}$ & $\begin{array}{l}\text { Doutorado em } \\
\text { Antropologia Social }\end{array}$ & $\begin{array}{l}\text { Secretaria de Estado de Educação de Mato } \\
\text { Grosso (SEDUC/MT) }\end{array}$ \\
\hline Guillermo Vega Sanabria & $\begin{array}{l}\text { Doutorado em } \\
\text { Antropologia Social }\end{array}$ & Universidade Federal da Bahia (UFBA) \\
\hline Íris Morais Araújo & $\begin{array}{l}\text { Doutorado em Ciência } \\
\text { Social (Antropologia } \\
\text { Social) }\end{array}$ & Universidade Estadual de Campinas (UNICAMP) \\
\hline Jacqueline Moraes Teixeira & $\begin{array}{l}\text { Doutorado em Ciência } \\
\text { Social (Antropologia } \\
\text { Social) }\end{array}$ & Universidade de São Paulo (USP) \\
\hline Janaína Ferreira Fernandes & $\begin{array}{l}\text { Doutorado em } \\
\text { Antropologia Social }\end{array}$ & Instituto Federal de Goiás (IFG) \\
\hline Laura Pérez Gil & $\begin{array}{l}\text { Doutorado em } \\
\text { Antropologia Social }\end{array}$ & Universidade Federal do Paraná (UFPR) \\
\hline $\begin{array}{l}\text { Leonardo Carbonieri } \\
\text { Campoy }\end{array}$ & $\begin{array}{l}\text { Doutorado em } \\
\text { Antropologia }\end{array}$ & $\begin{array}{l}\text { Pontifícia Universidade Católica do Paraná } \\
\text { (PUC/PR) }\end{array}$ \\
\hline $\begin{array}{l}\text { Liliana de Mendonça } \\
\text { Porto }\end{array}$ & $\begin{array}{l}\text { Doutorado em } \\
\text { Antropologia Social }\end{array}$ & Universidade Federal do Paraná (UFPR) \\
\hline Luciana de Oliveira Dias & $\begin{array}{l}\text { Doutorado em Ciências } \\
\text { Sociais }\end{array}$ & Universidade Federal de Goiás (UFG) \\
\hline
\end{tabular}




\begin{tabular}{|c|c|c|}
\hline Luciana Hartmann & $\begin{array}{l}\text { Doutorado em } \\
\text { Antropologia Social }\end{array}$ & Universidade de Brasília (UnB) \\
\hline $\begin{array}{l}\text { Maria Claudia Pereira } \\
\text { Coelho }\end{array}$ & Doutorado em Sociologia & $\begin{array}{l}\text { Universidade do Estado do Rio de Janeiro } \\
\text { (UERJ) }\end{array}$ \\
\hline Maria Elisa Maximo & $\begin{array}{l}\text { Doutorado em } \\
\text { Antropologia Social }\end{array}$ & $\begin{array}{l}\text { Instituto Superior e Centro Educacional Luterano } \\
\text { Bom Jesus (Ielusc) }\end{array}$ \\
\hline Maria Inês S. Borges & $\begin{array}{l}\text { Doutorado em } \\
\text { Antropologia Social }\end{array}$ & Universidade Federal do Paraná (UFPR) \\
\hline $\begin{array}{l}\text { Márcia L. Anacleto de } \\
\text { Souza }\end{array}$ & Doutorado em Educação & Prefeitura Municipal de Campinas \\
\hline Marília Sene de Lourenço & $\begin{array}{l}\text { Doutorado em } \\
\text { Antropologia Social }\end{array}$ & Instituto Federal do Paraná (IFPR) \\
\hline Nadège Mézié & $\begin{array}{l}\text { Doutorado em } \\
\text { Antropologia }\end{array}$ & Universidade Estadual de Campinas (UNICAMP) \\
\hline Nalayne Mendonça Pinto & Doutorado em Sociologia & $\begin{array}{l}\text { Universidade Federal Rural do Rio de Janeiro } \\
\text { (UFRRJ) }\end{array}$ \\
\hline $\begin{array}{l}\text { Nicolau Dela Bandera } \\
\text { Arco Netto }\end{array}$ & $\begin{array}{l}\text { Doutorado em Ciência } \\
\text { Social (Antropologia } \\
\text { Social) }\end{array}$ & Colégio Pedro II \\
\hline Paulo R. Homem de Góes & $\begin{array}{l}\text { Doutorado em } \\
\text { Antropologia Social }\end{array}$ & $\begin{array}{l}\text { Ministério Público do Estado do Paraná (MP/ } \\
\mathrm{PR} \text { - Grupo de Trabalho sobre a presença } \\
\text { indígena no Litoral/ Pontifícia Universidade } \\
\text { Católica do Paraná (PUC/PR) }\end{array}$ \\
\hline Patrícia Carvalho Rosa & $\begin{array}{l}\text { Doutorado em } \\
\text { Antropologia Social }\end{array}$ & $\begin{array}{l}\text { Instituto de Desenvolvimento Sustentável } \\
\text { Mamirauá }\end{array}$ \\
\hline Roberta Bivar C. Campos & $\begin{array}{l}\text { Doutorado em } \\
\text { Antropologia Social }\end{array}$ & Universidade Federal de Pernambuco (UFPE) \\
\hline $\begin{array}{l}\text { Rodrigo P. da R. } \\
\text { Rosistolato }\end{array}$ & $\begin{array}{l}\text { Doutorado em } \\
\text { Antropologia Social }\end{array}$ & Universidade Federal do Rio de Janeiro (UFRJ) \\
\hline $\begin{array}{l}\text { Ronaldo de Oliveira } \\
\text { Corrêa }\end{array}$ & $\begin{array}{l}\text { Doutorado em Ciências } \\
\text { Humanas }\end{array}$ & Universidade Federal do Paraná (UFPR) \\
\hline Rosane Aparecida Rubert & $\begin{array}{l}\text { Doutorado em } \\
\text { Desenvolvimento Rural }\end{array}$ & Universidade Federal de Pelotas (UFPel) \\
\hline Rossana Godoy Lenz & Doutorado em Educação & Universidad de La Serena \\
\hline Stella Zagatto Paterniani & $\begin{array}{l}\text { Doutorado em } \\
\text { Antropologia Social }\end{array}$ & Instituto Tricontinental de Pesquisa Social \\
\hline Tania Dauster M. e Silva & $\begin{array}{l}\text { Doutorado em } \\
\text { Antropologia Social }\end{array}$ & $\begin{array}{l}\text { Pontifícia Universidade Católica do Rio de Janeiro } \\
\text { (PUC-RJ) }\end{array}$ \\
\hline
\end{tabular}




\begin{tabular}{l|l|l}
\hline Tânia Welter & $\begin{array}{l}\text { Doutorado em } \\
\text { Antropologia Social }\end{array}$ & Universidade Federal de Santa Catarina (UFSC) \\
\hline Vanessa Petró & Doutorado em Sociologia & $\begin{array}{l}\text { Instituto Federal de Educação, Ciência e } \\
\text { Tecnologia do Rio Grande do Sul (IFRS) - } \\
\text { Campus Feliz }\end{array}$ \\
\hline Vitor Grunvald & $\begin{array}{l}\text { Doutorado em } \\
\text { Antropologia Social }\end{array}$ & $\begin{array}{l}\text { Universidade Federal do Rio Grande do Sul } \\
\text { (UFRGS) }\end{array}$ \\
\hline
\end{tabular}

